\title{
ESTADO ACTUAL DEL MEJORAMIENTO DEL ARROZ MEDIANTE LA UTILIZACIÓN DE ESPECIES SILVESTRES DE ARROZ EN CIAT ${ }^{1}$
}

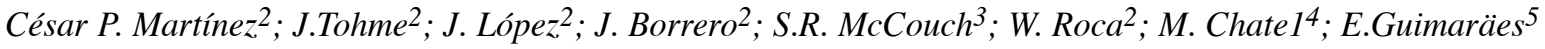

\begin{abstract}
RESUMEN
Estado actual del mejoramiento del arroz mediante la utilización de especies silvestres de arroz en CIAT. Este trabajo presenta el uso de especies silvestres de arroz en la obtención de germoplasma mejorado con mayor potencial de rendimiento y una base genética ampliada. Tres especies silvestres ( $O$. rufipogon, $O$. glaberrima y $O$. barthii) y 12 variedades mejoradas (padres recurrentes) fueron selecciona-das para desarrollar poblaciones a las cuales transferir selec-tivamente genes que controlan características de gran valor agronómico mediante el empleo de marcadores moleculares; las 36 poblaciones resultantes de los retrocruzamientos se encuentran en estados diferentes de desarrollo y se les aplicó selección fenotípica negativa contra caracteres agronómicos indeseables aportados por los progenitores silvestres. Se evaluaron 220-300 familias BC2F2 de cruzamientos específicos en ensayos replicados de rendimiento en varios sitios en Colombia. Segregación transgresiva para rendimiento $(\mathrm{kg} / \mathrm{ha})$ se detectó en la población Bg90-2*3/0. rufipogon y varias familias rindieron entre 5 y $15 \%$ más que $\mathrm{Bg} 90-2$; segregación transgresiva para resistencia al virus de la hoja blanca se detectó en la población O. Llanos $5 * 3 / 0$. rufipogon. Los datos preliminares sugieren que la introgresión de ciertos genes específicos presentes en $O$. rufipogon puede in-crementar el rendimiento en variedades mejoradas de arroz
\end{abstract}

\begin{abstract}
Current status of rice improvement through use of wild rice species at CIAT. This paper discusses the use of wild rice species to obtain an improved germplasm with a higher yield potential and a wider genetic base. Three wild species (O. rufipogon, O. glaberrima and O. barthii), and 12 improved varieties (recurrent parents) were selected to develop populations into which genes that control valuable agronomic cahracteristics are selectively transferred by the use of molecular markers. The 36 resulting populations from specific backcross schemes are now in different developmental phases, and underwent negative phenotypic selection against undesirable agronomic traits contributed by the wild parents. Between 220 and 300 BC2F2 families from specific crosses in replicated yield trials in severallocations in Colombia were evaluated. Transgressivesegregation for yield $(\mathrm{kg} / \mathrm{ha})$ was detected in the BG90-2*3/0 rufipogon population, and several families yielded between 5 and $15 \%$ more than the BG-90-2; transgressive segregation for resistance to the white leaf virus was detected in the O. llanos $5 * 3 / 0$ rufipogon population. Preliminary data sugests that introgression of certain genes from $O$. rufipogon may contribute to yield increase in improved rice varieties.
\end{abstract}

\section{INTRODUCCIÓN}

Alrededor de 148 millones de hectáreas de arroz se siembran a nivel mundial. Este grano constituye la base nutricional para gran parte de la población mundial; a nivel global el arroz proporciona $20 \%$ de energía y $15 \%$ de prostema per cápita. Se estima que para el año 2025 la población será de unas 8,3 billones de personas y que el 50\% de ellas consumirá arroz. Por ello, la producción actual de arroz del mundo (aproximadamente 575 millones de toneladas) debe aumentarse en $70 \%$ para responder a esa demanda.

Más del 90\% del arroz en el mundo se cultiva y se consume en Asia, mientras que la producción de América Latina (AL) representa el 3,5\% del total; más del

\footnotetext{
1 Presentado en la XLIII Reunión Anual del PCCMCA, Panamá 1997.

2 CIAT. Programa Arroz y Unidad de Investigación en Biotecnología. A.A. 6713. Cali,V. Colombia

3 Comell University. Plant Breeding. Dept. 252 Emerson Hall. Ithaca, N.Y4.

4 CIRAD-CA-CIAT. AA 6713. Cali, V. Colombia

5 CNPAF. C.P. 179 CEP74001-970. Goiania. Goias. Brazil.
} 
$70 \%$ de la producción del cereal en América Latina proviene de ecosistemas de riego y de tierras bajas. Esta producción aumentó de 9,9 a 18,8 millones de toneladas de 1966 a 1994; las variedades semienanas modernas combinadas con prácticas apropiadas de manejo permitieron en $\mathrm{AL}$ un incremento del rendimiento de $75 \%$ en los sectores de riego y tierras bajas (2,5 a $4,4 \mathrm{t} / \mathrm{ha})$.

El rápido crecimiento de la población en Asia y América Latina ejerce gran presión sobre los sistemas de producción de alimentos, los cuales ya enfrentan dificultades en estas regiones, especialmente en Asia. Para el año 2010 se predice un déficit de alimentos de 100 millones de toneladas por año, a menos que la comunidad agrícola mundial pueda desarrollar variedades y sistemas de cultivo que produzcan mucho más de lo que en la actualidad producen. Además, la viabilidad a largo plazo de nuestro sistema agrícola y el recurso base del que depende debe protegerse. Se requieren nuevos modelos de cooperación internacional, regional e interinstitucional y se hacen necesarias nuevas estrategias para el mejoramiento de las especies vegetales. Deben desarrollarse variedades altamente productivas, de buena calidad, tolerantes a las principales fuentes estrés bióticos y abióticos y eficientes en el aprovechamiento de los recursos. Los esfuerzos actuales en fitomejoramiento deben modernizarse utilizando una combinación de los enfoques clásicos y los de biotecnología. Para aquellos que están involucrados con la investigación agrícola cada vez será más esencial estar familiarizados con gran variedad de enfoques y métodos de mejoramiento genético del cultivo. La aplicación efectiva de la biotecnología al mejoramiento genético de los cultivos, requiere de la existencia de programas de mejoramiento bien establecidos y de su adaptación al uso de la biotecnología. A pesar de haberse avanzado en el desarrollo de la capacidad biotecnológica molecular del arroz, su integración práctica en el fitomejoramiento ha sido muy limitada.

\section{Variación genética estrecha y la barrera del rendi- miento}

Los recursos genéticos vegetales constituyen la fuente imprescindible de genes para el mejoramiento de los cultivos. Mediante la recombinaciónde genes favorables, los fitomejoradores han podido incrementar la productividad de los cultivos, mejorar su calidad y reducir los costos de producción. Hasta ahora, los programas de fitomejoramiento tradicionales sólo han explotado exitosamente un porcentaje muy limitado de la variabilidad genética existente en una especie dada.

Todas las especies vegetales fueron domesticadas originalmente por el hombre a partir de especies sil- vestres, un proceso que intrínsecamente redujo la variabilidad genética (Simmonds, 1976; Ladizinsky, 1985; Tanskley; Nelson, 1996). El mejoramiento intensivo moderno redujo aún mas dicha variabilidad, problema mas crítico en especies que se autofecundan como es el caso del arroz (Wang; Second; Tanksley, 1992).

La variabilidad genética estrecha existente en las variedades mejoradas modernas las volvió más vulnerables a estreses bióticos y abióticos, lo cual podría explicar la tasa más lenta de progreso genético alcanzado últimamente por los mejoradores (Tanksley; Nelson 1996). Este problema es especialmente crítico cuando se relaciona con el rendimiento y ha culminado en el fenómeno conocido como "barrera de rendimiento."

Existe una necesidad urgente de aumentar la producción de arroz de manera sostenible pero la variación genética reducida actúa en contra de ella. En América Latina, el mejoramiento del arroz de riego ha dependido de un núcleo genético principal compuesto por $12 \mathrm{cul}$ tivares (Cuevas-Pérez et al. 1992). Numerosos estudios indican que el potencial de rendimiento per se de arroz de riego en América Latina ha alcanzado un plateau (Plan de mediano plazo del CIAT 1993-1998). En Colombia el promedio de rendimiento del arroz de riego no ha variado desde 1970 (Fedearroz, 1993). En el IRRI, el último avance mayor en potencial de rendimiento del arroz se obtuvo con el desarrollo de IR8; sin embargo, en los últimos 30 años, los esfuerzos de mejoramiento se han dedicado ampliamente a mejorar la resistencia a plagas, enfermedades, calidad y adaptación general pero el rendimiento se mantiene en niveles más o menos constantes. Se ha desarrollado un nuevo tipo de planta en IRRI (Peng; Khush; Cassman, 1994) con un potencial de rendimiento mayor; sin embargo, no está listo aún para siembra comercial y requiere un mejoramiento adicional en varias características relevantes.

Por fortuna, la variación genética es abundante en la naturaleza; las especies silvestres y los cultivares tradicionales se pueden encontrar aún in situ y en bancos de germoplasma. Según Tanksley; Nelson (1996), los fitomejoradores no han podido explotar la mayoría de este potencial genético debido a dos razones: i) dificultad en la identificación de genes para aumentar el rendimiento y mejorar la calidad en el germoplasma silvestre y ii) ligamiento estrecho entre características deseables e indeseables. Se necesita una estrategia mas eficiente para facilitar la transferencia selectiva de genes "deseables", sin transferir los numerosos genes "indeseables" que se encuentran en especies silvestres. En él caso del arroz (Vaughan; Sitch. 1991), el género Ory$z a$ cuenta con aproximadamente 20 especies silvestres y dos especies cultivadas (O. sativa y $O$. glaberrima). Algunas de estas especies silvestres se han utilizado en 
programas de mejoramiento, en especial en el IRRI, pero en la mayoría de los casos se han utilizado como fuentes de genes para resistencia a enfermedades e insectos. Datos recientes (Xiao et al, 1996) sugieren que algunas especies silvestres de arroz poseen genes capaces de aumentar el rendimiento y mejorar la calidad de las variedades modernas. Por otra parte, se encuentran disponibles dos mapas moleculares de arroz bien saturados (Causse et al., 1994; Kurata et al., 1994). Estos mapas contienen loci codominantes estrechamente ligados que se pueden monitorear para asociarlos a genes que controlan características de importancia agronómica (Tanksley et al., 1989; Paterson; Tanksley; Sorrell, 1991). Si estos mapas se utilizan con métodos tradicionales de mejoramiento, pueden ayudar a los mejoradores a localizar y a transferir selectivamente genes involucrados en rendimiento, calidad y adaptabilidad a diferentes limitantes de la producción (Xiao et al., 1995; Ahn; Bollich; Tanksley, 1992; Nakamura et al., 1994; McCouch; Doerge, 1995). Recientemente Tanksley; Nelson, (1996) propusieron un método llamado "Análisis avanzado de QTL usando Retrocruzamiento", el cual combina el análisis QTL con el desarrollo de variedades. Los fitomejoradores disponen de una herramienta poderosa para mejorar sus acervos genéticos. El propósito de este trabajo es presentar el estado de la hibridación interespecífica en arroz en CIAT.

\section{MATERIALES Y MÉTODOS}

\section{DESARROLLO DE LAS POBLACIONES}

Unas pocas plantas (2-3) de cada especie silvestre (O. rufipogon, $O$. glaberrima, y $O$. barthii) se hibridizaron a varias plantas de cada uno de los cultivares mejorados (padre recurrente) que se incluyen en el Cuadro 1. El padre recurrente se escogió de acuerdo a ciertas características específicas. Se obtuvieron cruces simples y se sembraron en el invernadero en junio de 1994 en CIAT-Palmira. Tres plantas F1 se retrocruzaron al cultivar mejorado, utilizando el último como el padre femenino; se obtuvieron aproximadamente 100-180 semillas BC1F1 por cada combinación. Las plantas BCIF1 resultantes se trasplantaron $(30 \times 50 \mathrm{~cm})$ y se evaluaron de acuerdo con sus características fenotípicas; se aplicó selección fenotípica negativa, por características agronómicas no deseables (tipo de planta abierto, desgrane excesivo, aristas largas, granos de color oscuro, alta esterilidad, etc.) para reducir la selección a los mejores individuos (40-50). Cada individuo BC1 seleccionado se retrocruzó de nuevo al padre recurrente y se obtuvieron entre 30-50 semillas $\mathrm{BC} 2 \mathrm{~F} 1$ por cruce; 20 semillas $\mathrm{BC} 2 \mathrm{~F} 1$ de cada planta $\mathrm{BC} 1$ seleccionada se sembraron en bandejas de plástico en el invernadero y más tarde se transplantaron $(30 \times 40 \mathrm{~cm})$ bajo

Cuadro 1. Materiales utilizados en la hibridación interespecífica en CIAT.

\begin{tabular}{|c|c|c|c|c|}
\hline Progenitor & Accesión No. & Fuente & Origen & Notas \\
\hline \multicolumn{5}{|l|}{ Donante } \\
\hline O. rufipogon & 105491 & IRRI & Malaysia & Ancestro de $O$. sativa \\
\hline O. glaberrima & 103544 & IRRI & Mali & Cultivado en Africa \\
\hline O. barthii & 104119 & IRRI & Chad & Pariente de $O$. glaberrima \\
\hline \multicolumn{5}{|c|}{ Recipiente (Padre Recurrente) } \\
\hline Cypress & & Louisiana & USA & Japónica Tropical, calidad \\
\hline Lemont & & Texas & USA & Japónica Tropical, calidad \\
\hline RU9403006 (Jefferson) & & Texas & USA & Japónica Tropical, calidad \\
\hline Oryzica Llanos 5 & & CIAT & Colombia & Indica; resistente a $P$. oryzae \\
\hline BG90-2 & & CIAT & Sri Lanka & Indica; alto rendimiento \\
\hline Morelos A88 & & CIAT & México & Buena habilidad combinatoria \\
\hline Oryzica 3 & & CIAT & Colombia & Indica; alto rendimiento \\
\hline O. sabana 6 & & CIAT & Colombia & Japónica Tropical; secano \\
\hline O. turipana 7 & & CIAT & Colombia & Japónica Tropical; secano \\
\hline Progreso & & CIAT & Brasil & Japónica Tropical; secano \\
\hline CAIAPO & & CIAT & Brasil & Japónica Tropical; secano \\
\hline CT6196-33-11-1-3 & & CIAT & Colombia & Japónica Tropical; secano \\
\hline
\end{tabular}


condiciones de riego. Se aplicó una selección fenotípica negativa de nuevo y se seleccionaron y cosecharon los mejores individuos para generar semilla BC2F2; aproximadamente se seleccionaron 220-300 plantas $\mathrm{BC} 2 \mathrm{Fl}$ por cada cruce para pruebas de campo. Cada planta $\mathrm{BC} 2 \mathrm{Fl}$ seleccionada y los progenitores se evaluaron para 12 características agronómicas que incluían días a floración y cosecha, altura de planta, número de macollas, longitud de panícula, espiguillas por planta, granos por panícula, peso de 1.000 granos y rendimiento por planta. Con base en observaciones de campo y potencial genético de las poblaciones se escogieron sólo tres poblaciones para pruebas de campo (Bg90-2/ O. rufipogon, O. Llanos 5/ O. rufipogon, y Caiapo/O. rufipogon). La Figura 1 resume el esquema seguido en el desarrollo de las poblaciones.

\section{Ensayos de campo (Familias BC2F2)}

Las 300 familias $\mathrm{BC} 2 \mathrm{~F} 2$ derivadas de los cruces Bg-90-2/ O.rufipogon y Caiapo/ O. rufipogon, y las 220 familias del cruce O. Llanos 5/ O. rufipogon se sembra- ron en ensayos de rendimiento replicados en cuatro sitios en Colombia (CIAT-Palmira y CIAT-Santa Rosa. Villavicencio, Est. Exp. La Libertad. Villavicencio, y Saldaña. Tolima). El cruce Caiapo/ O. rufipogon se sembró bajo condiciones de secano en suelos ácidos y las otras bajo condiciones de riego y secano favorecido (Santa Rosa). En CIAT Palmira se utilizó el transplante (20x30cm), mientras que en los otros sitios se utilizó la siembra directa en surcos. Se utilizó un diseño completamente al azar con dos repeticiones, y parcelas de dos surcos de cinco metros de longitud. Se tomaron datos de las 12 características agronómicas descritas antes y se adicionó el rendimiento/parcela. Se siguieron las prácticas de cultivo recomendadas para cada sitio; sin embargo, en Santa Rosa y la Libertad los ensayos se protegieron con aplicaciones de fungicidas para evaluar mejor el potencial de rendimiento. Las tres poblaciones también se evaluaron por su resistencia a Tagosodes y al virus de la hoja blanca en condiciones de invernadero en CIAT. Palmira y por resistencia a $P$. oryzae en camas de infección en Santa Rosa.
BG90-2/O. rufipogon

O. LLANOS 5/O. rufipogon

CAIAPO/O. rufipogon

Caracterización molecular:

Progenitores, F1: Hecho

Familias BC2F2: En Progreso

Por hacer:

Análisis QTL -> Identificar familias con

Alelos intereses $->$ 3er. BC $->$ Desarrollo

de líneas isogénicas $->$ Var.
100-180 BC1F1 semillas

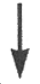

Selección negativa

caracteres indeseables

Identificación fenotípica

mejores plantas (40-50)<smiles>[C+]1[CH]C1</smiles>

BC2F1: 900-1000 semillas<smiles>C1C[C@H]2CC12</smiles>

Selección negativa caracteres indeseables<smiles>C[Al]</smiles>

Seleccionar $\pm \mathbf{3 0 0}$ familias BC2F2

Ensayo replicado rendimiento

(varios sitios)

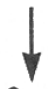

Parcelas: 2 surcos/fam.;

5m.; 2 rep.

(competencia malezas)

Tomar datos agronómicos (12 caracteres)

Fig. 1. Esquema seguido en CIAT para el desarrollo de las poblaciones. 


\section{Caracterización molecular}

Hojas frescas de arroz fueron maceradas en nitrógeno líquido para lograr una fragmentación celular y luego se extrajo el ADN siguiendo la metodología propuesta por McCouch et al, (1988) y modificada en CIAT, (1995). Para la aplicación de los marcadores RFLP's se prepararon filtros de ADN (4mg/línea) que contenían: $O$. rufipogon; O. glaberrima; $O$. barthii; $\mathrm{O}$. Llanos 5; O. Sabana 6; Fanny; Caiapo; Bg90-2 y los correspondientes a las F1's. Dichos parentales fueron digeridos con las enzimas de restricción DraI, EcoRI, EcoRV y HindIII.

A partir de los mapas moleculares de arroz desarrollados en la Universidad de Comell por McCouch, Paralelamente a dichos marcadores se están utilizando marcadores microsatélites para saturar aquellas regiones del genoma que no resultan polimórficas por RFLP's; se busca integrar estos dos tipos de marcadores y hacer mas fácil la ubicación de caracteres asociados a los QTL's.

\section{RESULTADOS Y DISCUSIÓN}

\section{Evaluación y selección de sondas}

Los datos indicaron que de un total de 140 dones evaluados, 90 fueron polimórficos (64\%); el polimorfismo fue mayor entre $O$. rufipogon y los cultivares japónica Fanny, O. Sabana 6 y Caiapo, comparado con el encontrado entre $\mathrm{O}$. rufipogon y los cultivares indica $\mathrm{O}$. Llanos 5 y Bg90-2. La evaluación de familias BC2F2 con dones RFLP polimórficos y microsatélites se está realizando.

\section{Desarrollo de la población}

Se hicieron 36 cruzamientos (Cuadro 2) utilizando las líneas parentales que se presentan en el Cuadro 1; el desarrollo de las poblaciones provenientes de estos cruces se está adelantando pero en diferentes etapas; las poblaciones más avanzadas han pasado por dos series de retrocruzamientos al padre recurrente. A pesar de que los arroces silvestres utilizados y el arroz cultivado pertenecen al mismo genoma AA y que por consiguiente la cruzabilidad entre ellos debe ser fácil, se presentaron problemas de esterilidad y de aborto de embriones en algunos casos, en especial en los cruzamientos con $O$. barthii. Los problemas con el desarrollo de embriones se observaron 10-15 días después de la polinización. Por lo tanto, el rescate del embrión resultó un éxito para solucionar el problema; las plantas híbridas recuperadas a través del rescate del embrión también segregaron por características deseables e indeseables y se utilizaron en el esquema de retrocruzamiento si tenían un fenotipo deseable.

\section{Ensayos de rendimiento}

Estas evaluaciones se efectuaron durante juniodiciembre de 1996. Aún se están procesando los datos tomados sobre las 12 características agronómicas importantes y sólo se presenta información preliminar generada en CIAT - Palmira. En la Figura 2 se observa la distribución del rendimiento de grano $(\mathrm{kg} / \mathrm{ha})$ de 38 familias BC2F2 del cruce Bg90-2/ O. rufipogon; estas familias fueron seleccionadas en el campo con base en fenotipo y los datos de las parcelas de rendimiento están constituidos por 40 plantas (20 plantas/surcos x 2 surcos) promediado sobre dos replicaciones. Se puede observar la segregación transgresiva, con algunas líneas presentando un rendimiento entre 5 y $15 \%$ más alto que el padre recurrente $\mathrm{Bg} 90-2$. Estos datos preliminares están de acuerdo con los provenientes del trabajo que se adelanta en China en el Hunan Hybrid Rice Research Center (Xiao et al, 1996; McCouch, 1995) y en Corea del Sur. Estos datos reportados por diferentes grupos que trabajan con padres recurrentes diversos pero que utilizaron los mismos donantes silvestres sugieren que la introgresión de ciertos genes de $O$. rufipogon puede contribuir positivamente al incremento de los rendimientos en cultivares élite de arroz, aún cuando $O$. $r u$ fipogon en sí mismo es inferior a Bg90-2. Aún más, los datos de China (McCouch, 1995) sugieren que dos QTL=s hallados.en los cromosomas uno y dos de $O$. rufipogon son responsables de los incrementos de rendimiento. Estos QTL's se han designado como yldl.l. y yld2.1 (Xiao et al 1996).

El análisis molecular y el de QTLs en progreso en CIAT van a indicar si estamos o no tratando con los mismos QTL=s reportados por el grupo de China; además, el análisis QTL que se realice en las poblaciones desarrolladas con $O$. glaberrima y $O$. barthii mostrará si estas especies poseen o no los mismos QTLs para rendimiento que se encontraron en $O$. rufipogon. $\mathrm{Si}$ cada especie silvestre posee alelos específicos positivos para rendimiento, entonces los mejoradores de arroz contarán con una herramienta muy valiosa para incrementar el rendimiento en una manera sistemática y piramidal, esto es, en un proceso por etapas.

Por otra parte, se evaluaron por su resistencia al virus de la hoja blanca 220 familias $\mathrm{BC} 2 \mathrm{~F} 2$ derivadas del cruce Oryzica Llanos 5/ O. rufipogon bajo condiciones de campo en CIAT-Palmira utilizando una colonia altamente virulenta de Tagosodes oryzicola. La distribución de la incidencia de la enfermedad (\%) con base en una escala de 0 - 9 ( $0=$ ningún síntoma de enfermedad; $9=>70 \%$ plantas enfermas) se presenta en la Figura 3. 
Cuadro 2. Cruces interespecíficos realizados entre varios cultivares de arroz de riego y de secano y tres especies silvestres de arroz.

\begin{tabular}{|c|c|}
\hline O. rufipogon & $\begin{array}{l}\text { BG90-2 II } 2 \text { x BG90-23 } \\
\text { MORELOS A88 // } 2 \text { x MORELOS A883 Oryzica } 3 \text { // } 2 \text { x Oryzica } 33 \\
\text { Oryzica llanos } 5 \text { // } 2 \text { x Oryzica llanos } 53 \text { LEMONT II LEMONT } \\
\text { O. rufipogon3 } \\
\text { RU94030006 // } 2 \text { x RU940300063 } \\
\text { Cypress // } 2 \text { x Cypress } 3 \\
\text { Oryzica sabana } 6 \text { // } 2 \text { x Oryzica sabana } 63 \\
\text { Oryzica turipana } 71 \\
\text { PROGRESSO1 } \\
\text { CT6196-33-11-1-3 } \\
\text { CAIAPO // } 2 \text { x CAIAP03 }\end{array}$ \\
\hline O. barthii & $\begin{array}{l}\text { BG90-2 II BG90-22 } \\
\text { MORELOS A88 // } 2 \text { x MORELOS A883 } \\
\text { Oryzica } 3 \text { // } 2 \text { x ORYZICA } 33 \\
\text { Oryzica llanos } 5 \text { // Oryzica llanos } 52 \\
\text { LEMONT // LEMONT2 } \\
\text { O. barthii//RU940300062 } \\
\text { Cypress // Cypress2 } \\
\text { Oryzica sabana } 6 \text { // Oryzica sabana } 62 \\
\text { Oryzica turipana } 7 \text { // Oryzica turipana } 72 \\
\text { PROGRESSO1 } \\
\text { // CT6196-33-11-1-32 } \\
\text { CAIAPO1 }\end{array}$ \\
\hline
\end{tabular}

BG90-2 // BG90-22

MORELOS A881

Oryzica 3 // Oryzica 32

Oryzica llanos 51

LEMONTl

O. glaberrima

O. glaberrimal

RU94030006 1/2 x RU940300063

Cypress1

Oryzica sabana 6 // Oryzica sabana 62

Oryzica turipana 7 // Oryzica turipana 72

PROGRESSO 1

CT6196-33-11-1-31

CAIAPOI

$1=$ Efectuados cruce simples.

2 = Efectuados primero retrocruzamientos; en algunos casos segundos retrocruzamientos en camino. 3 = Efectuados segundos retrocruzamientos; en algunos casos ensayos de rendimientos replicados con progenies F2BC2 en camino. 


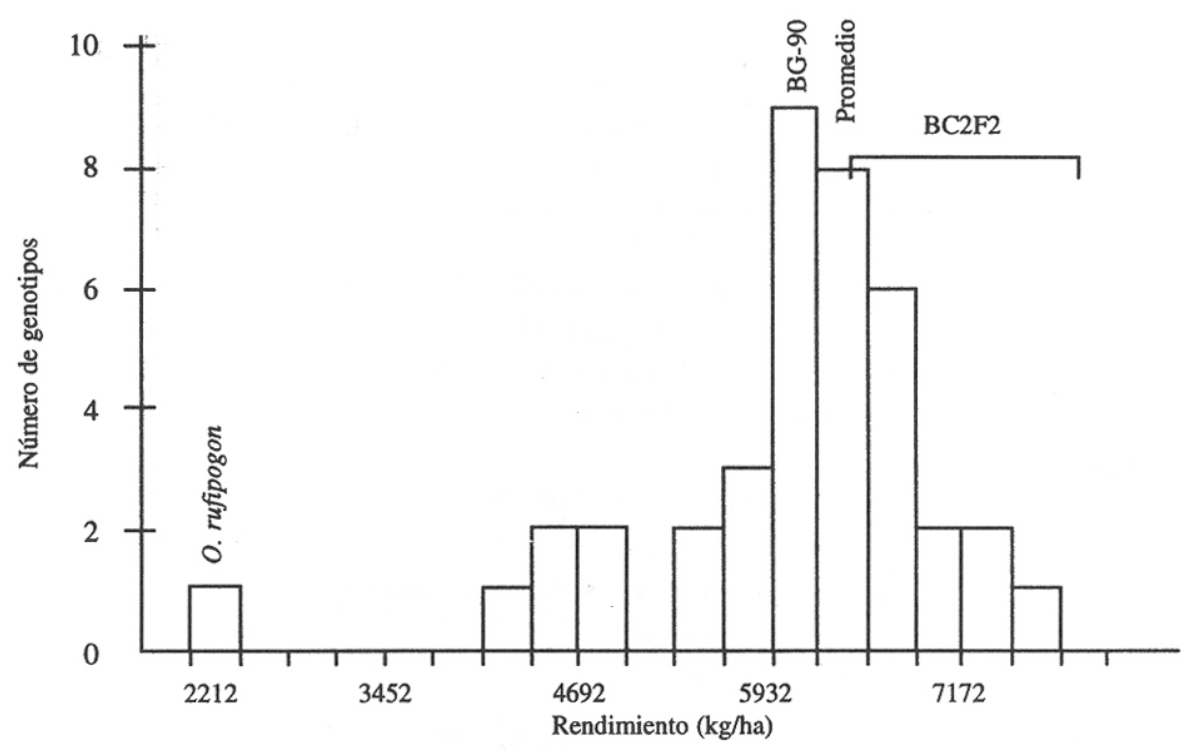

Fig. 2. Distribución del rendimiento en familias BC2F2 del cruzamiento interespecífico BG902/0. rufipogon.

La segregación transgresiva para la resistencia al Virus de la Hoja Blanca se puede observar, con aproximadamente $50 \%$ de las familias ubicadas en las categorías uno y tres; los progenitores O. Llanos 5, yO. rufipogon cayeron en las categorías cinco y nueve, respectivamente. Estos datos preliminares de un experimento sin replicar parecen sugerir que existen alelos positivos para el virus de la hoja blanca en $O$. rufipogon los cuales podían contribuir a un incremento en la resistencia a esta enfermedad. Se necesitan más pruebas pa- ra confirmar este resultado y determinar el tipo de acción génica presente.

En resumen, los datos preliminares corroboran la hipótesis que la introgresión de ciertos alelos específicos de $O$. rufipogon puede contribuir positivamente no sólo para incrementar el rendimiento en cultivares mejorados de arroz sino también en términos de resistencia a estrés. Esta información también proporciona la base para implementar el método propuesto por

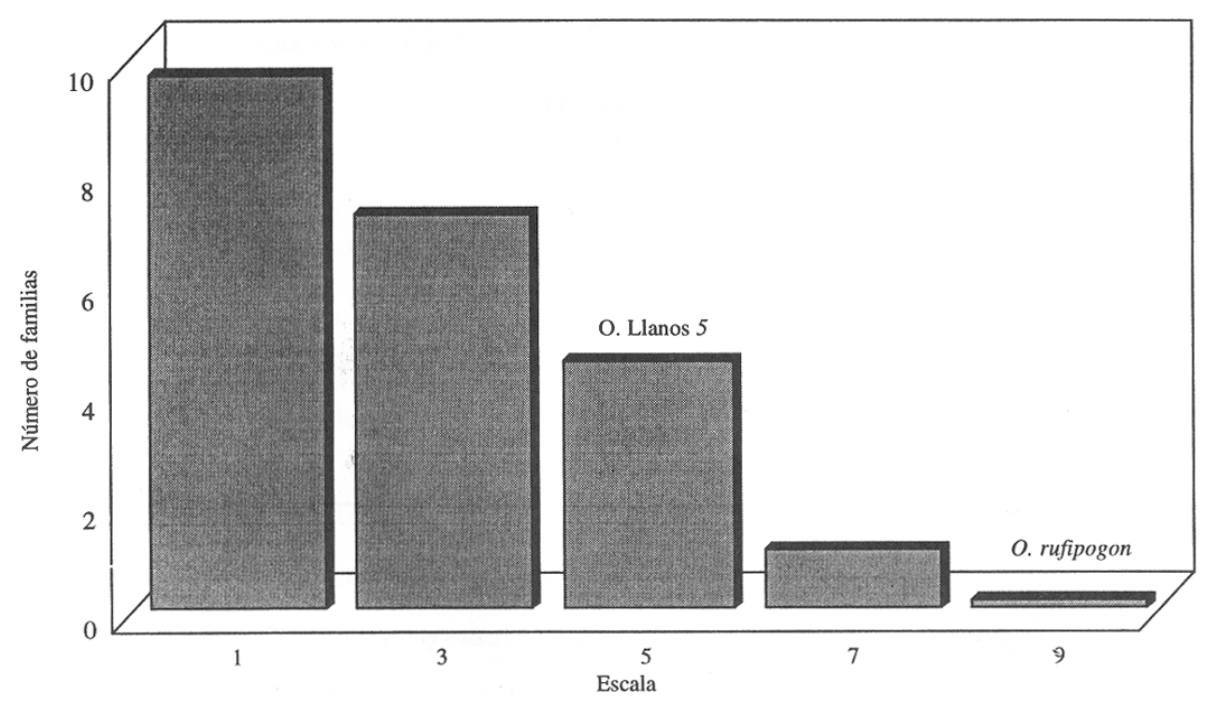

Fig. 3. Distribución de clases de la reacción al virus de la hoja blanca en la generación F2RC2 del cruce Oryzica Llanos 5/0. rufipogon. 
Tanksley; Nelson, (1996) referido como Análisis Avanzado QTL usando retro cruzamientos para el descubrimiento simultáneo y la transferencia de QTLs valiosos presentes en el germoplasma silvestre a líneas élite de arroz.

\section{LITERATURA CITADA}

AHN, S.; BOLUCH C. N.; TANKSLEY S. D. 1992. RFLP tagging of gene for aroma in rice. Theor. Appl. Genet. 84: 825-828.

CAUSSE M; FULTON T. M.; CHO Y. G.; AHN S. N.; WU K; XIAO J.; CHUNWONGSE J; YU Z.; RONALD, P. c.; HARRINGTON S. B.; SECOND G. A; MCCOUCH S. R.; TANKSLEY S. D. 1994. Satured molecular map of the rice genome based on an interspecific backcross population. Genetics 138: 1251-1274.

CIAT. 1995. Protocolos para marcadores moleculares. Unidad de biotecnología.

CUEVAS-PJREZ, F., E. P. GUIMARNES, L. E. BERRIO, AND D. 1. GONZÁLEZ. 1992. Genetic base of irrigated rice in Latin America and the Caribbean. Crop Sci. 32:1054-1059

FEDEARROZ. 1993. Arroz en Colombia, 1980-1993. Santa Fe de Bogotá, D.C. Federación Nacional de Arroceros. 88p.

KURATAN; NAGAMURA Y; YAMAMOTO K; HARUSHIMA Y; SUE N; WU J; SHOMURA A; SHIMIZU T; UN S. Y; INOUE T; FAKUDAA; SHIMANO T; KU-BOKI Y; TOYAMA T; MIYAMOTO Y; KIRIHARA T; HAYASAKA K; MIYAO A; MONNA L; ZHONG H.S.; TAMURA Y; WANG Z. X.; MOMMA Y; UMEHARA Y; YANO M; SASAKI T; MINOBE Y. 1994. A 300 kilobase interval genetic map of rice including 883 expressed sequences. Nature Genetics 8: 365-372.

LADIZINSKY, G. 1985. Founder effect in crop-plant evolution. Econ. Bot. 39(2): 191- 199.

McCOUCH, S.R.; KOCHERT, G.; YU, Z.H.; WANG, Z.Y; KHUSH, G.S.; COAFMAN, w.R. 1988. Molecular mapping of rice chromosomes. Theor. Appl. Genet. $76: 815-829$.

McCOUCH S. R. 1995. Marker - assisted discovery and transfer of "wild QTLs" into elite rice germplasm. Proyect submitted to USDA for funding. Cornell University. Plant Breeding Dept. Ithaca, N.Y 18p.

McCOUCH S. R; DOERGE R. W. 1995. QTL mapping in rice. Trends in Genet (11:482-487).
NAGAMURA, Y. 1993. Ahigh density STS and EST linkage map of rice. Rice Genome 2(1):3.

NAKAMURA Y.; NAGAMURAY; MURATAN.; MINOBE Y. 1994. Linkage localization. of the starch branching enzyme 1 (Q enzyme 1) gene in rice. Theor. Appl. Genet. 89:859-860.

PATERSON, A H.; TANKSLEY S. D.; SORRELL M. E. 1991. DNA markers in plant improvement. In: Advances in Agronomy (Sparks, D.L., ed.), Academic Press Inc., New York. pp. 39-90.

PENG, S.; KHUSH G. S.; K G. CASSMAN. 1994. Evolution of the new plant ideotype for increased yield potential. In: Cassman, K G. (ed).: Breaking the yield barrier. Proc. Workshop on Rice Yield Potential in favorable. Environments. IRRI, 29 Nov-04 Dec. 1993. Intern. Rice Res. Insto Manila-Philippines. pp. 5-20.

SAITO, A.; YANO M.; KISHIMOTO N.; NAKAGAHRA M.; YOSHIMURAA.; SAITO K 1991. Linkage Map of Restriction fragment length polymorphism loci in rice. Japanese Journal of Breeding 41:665-670.

SIMMONS, NW. 1976. Evolution of crop plants. Longman, London, New York.

TANKSLEY, S. D.; YOUNG N. D.; PATERSON A. H.; BONIERBALE M. W. 1989. RFLP mapping in plant breeding: New tools for an old science. Biotechnology 7: $257-264$

TANKSLEY S. D.; NELSON J. C. 1996. Advanced backcross QTL analysis: a method for the simultaneous discovery and transfer of valuable QTLs from unadapted germplasm into elite breeding lines. Theor Appl Genet 92: 191-203.

VAUGHAN, D. A; SITCH L. A 1991. Gene flow from the jungle to farmers. Bioscience 41: 22-28.

XIAO, J.; U J.; YUAN L.; TANKSLEY S. D.. 1995. Dominance is the major genetic basis of heterosis in rice as revealed by QTL. analysis using molecular markers. Genetics 140: 745-754.

XIAO, 1.; GRANDILLO S.; AHN S.N.; McCOUCH SR; TANKSLEY S.D. 1996. Genes from wild rice improve yield. Nature. 184:223-224.

WANG, Z. w.; SECOND G.; TANKSLEY S. D. 1992. Polymorphism and phylogenetic relationships among species in the genus Oryza as deterrnined by analysis of nuclear RFLPs. Theor. Appl. Genet. 83:565-581. 